DOI: https://doi.org/10.11144/Javeriana.umed58-2.reum

\title{
Enfermedades reumáticas en Colombia: quince años de investigaciones psicológicas ${ }^{*}$
}

\section{Rheumatic Diseases in Colombia. Fitteen Years of Psychological} Research

Fecha de recepción: 03/08/2016 | Fecha de aprobación: 09/05/2017 | 05/01/2017

\author{
César Andrés Gómez-Acosta \\ Fundación Universitaria Sanitas, Colombia \\ Stefano Vinaccia Alpi \\ Fundación Universitaria Sanitas, Colombia \\ Japcy Margarita Quiceno \\ Universidad de Medellín, Colombia
}

Cómo citar: Gómez-Acosta CA, Vinaccia Alpi S, Quiceno JM. Enfermedades reumáticas en Colombia: quince años de investigaciones psicológicas. Univ Med. 2017;58(2):1-11. doi: https://doi.org/10.11144/ Ja veriana.umed58-2.reum

\section{RESUMEN}

El objetivo de esta revisión es analizar las principales investigaciones psicosociales que se han llevado a cabo en Colombia con pacientes reumáticos. Por un lado, se evalúan los estudios desarrollados con pacientes de artritis reumatoide, lupus eritematoso sistémico y fibromialgia; por otro, se analizan los procesos de evaluación y de intervención que se han desarrollado en el área. Se le da relevancia a la descripción de los resultados alcanzados en cada estudio. Por último, se analizan los resultados encontrados en las investigaciones colombianas.

Palabras clave

enfermedades reumáticas; investigación psicológica; Colombia.

\section{ABSTRACT}

The purpose of this review is to present an analysis of the main psychosocial research that has been conducted in Colombia with rheumatic patients. First, developed studies were evaluated in rheumatoid arthritis, systemic lupus erythematous and fibromyalgia patients. Then taken into account other assessment processes and interventions that are developed in the area, with special emphasis on the description of the results achieved in each study. Finally, an analysis of the results found in Colombian research is done.

Keywords

rheumatic diseases; psychological research; Colombia.

\section{Introducción}

Las enfermedades reumáticas corresponden a un grupo de enfermedades crónicas autoinmunes, multifactoriales, que afectan en particular el tejido músculo-esquelético y conectivo, que lo deforman y lo hacen disfuncional [1,2]. Típicamente, son enfermedades sistémicas [3] que, a menudo, involucran síntomas como dolor crónico, pérdida sistemática de la motricidad y fatiga articular y muscular 
[4]. Dichas enfermedades llegan a afectar de manera significativa el sistema nervioso central (induciendo cambios en la personalidad y el rendimiento cognitivo) y el sistema nervioso periférico (induciendo neuropatías específicas) [3]. Dentro de este conjunto de patologías se encuentran la artritis reumatoide, la fibromialgia, el lupus eritomatoso sistémico, la artrosis $\mathrm{u}$ osteoartritis (reumatoide e infecciosa), la espondilitis anquilosante, la gota, entre otras.

En este tipo de enfermedades cobran importancia las representaciones mentales y las emociones que hacen referencia a las características, etiquetas y causas que se le atribuyen tanto al trastorno en sí como al tratamiento y sus consecuencias posteriores [5]. Así mismo, en dichos trastornos es frecuente que se aumente el riesgo de desarrollar tanto ansiedad (expresado fundamentalmente a partir de conductas evitativas) como depresión (expresada con sensaciones de tristeza e infelicidad recurrentes) [6].

En varias regiones del mundo se ha desarrollado un abordaje importante acerca de los factores psicosociales y riesgos en salud mental asociados a este tipo de enfermedades; $\sin$ embargo, en contraste con otras enfermedades crónicas [7], en Colombia solo hasta hace quince años se han venido relacionando aproximaciones específicas hacia temas como la calidad de vida, la resiliencia, el afrontamiento, los tipos de personalidad y la presencia de condiciones de salud mental, como depresión y estrés.

\section{Objetivos}

En primer lugar, presentar un análisis acerca de los estudios colombianos desarrollados en los últimos quince años donde se describan los factores psicosociales que más comúnmente se ven afectados en dichas condiciones somáticas. $\mathrm{Y}$, en segundo lugar, discutir los hallazgos para proyectar posibles abordajes investigativos y terapéuticos futuros en el área.

\section{Materiales y método}

Se abordaron 51 artículos indexados procedentes de las bases de datos Medline, PsycARTICLES, Science Direct y Springer Journal, así como dos capítulos de libros, en la metodología propia de investigación documental.

\section{Resultados}

Se realizará en primera instancia la presentación de las generalidades de cada síndrome, seguidas de los comentarios a los estudios que relacionan dicha patología con algunos factores psicosociales, señalando que es notoria la diferencia entre la cantidad de estudios que abordan el tema de artritis reumatoide con relación a los trabajos que se refieren a otras patologías reumáticas.

\section{Artritis reumatoide}

La artritis reumatoide afecta principalmente la membrana sinovial de las articulaciones, en una relación de 3:1 a las mujeres, y aumenta de prevalencia conforme sube la edad de los pacientes [4]. Sus principales síntomas son inflamación, aumento del calor, rigidez, fatiga y cansancio en las áreas afectadas [8]. En esta patología se observa un estado de pasividad y desesperanza, dada por la falta de contingencia entre la conducta del sujeto y las consecuencias [9], de manera tal que el propio individuo no tiene el control del entorno. En ese caso, un diagnóstico de artritis reumatoide actúa como una condición amenazante e implica una serie de adaptaciones a nuevas situaciones, algunas poco predecibles. Al ser una condición progresiva, puede generar sensaciones de inseguridad y de reducción de la autoeficacia.

Un primer abordaje empírico demostró con 79 pacientes que en estas personas se presentan síntomas de depresión, discapacidad, infelicidad, dolor, baja percepción de autoeficacia y de calidad de vida [10], independiente de variables como la edad, el sexo o el tiempo de 
diagnóstico de la enfermedad; no obstante, dicha condición somática afecta de manera significativa la salud mental de quienes la padecen. Posteriormente, otro estudio señala que las variables sociodemográficas inciden en la forma como los pacientes asumen su situación médica y ponderan su calidad de vida [11]; así mismo, ellos presentaron bajos índices en la incapacidad funcional y en desesperanza aprendida, lo cual relacionan con el amplio apoyo económico, afectivo y social que es brindado por sus familiares y amistades, aspecto que contrasta con los resultados de otras latitudes, donde se evidencian fuertes correlaciones entre incapacidad funcional, dolor y desesperanza.

Esta condición también es evidente en aquellos pacientes que desarrollan problemas de locomoción por tener pie reumatoide, específicamente movimientos subtalares y tibiotalares, así como restricciones en la plantiflexión [12]. En esta última investigación se reconoce que la duración del trastorno aumenta la correlación entre el daño y la discapacidad demostrada, elementos que, a su vez, se relacionan con mayor dolor percibido y con un deterioro en la calidad de vida relacionada con su salud.

En otra investigación, al aplicar la versión española del Quality of Life Measure for Rheumatoid Arthritis a 82 sujetos colombianos se encontraron niveles moderados en las áreas de desempeño emocional, salud mental, desempeño social y vitalidad; no obstante, se reportaron cifras bajas en las áreas de capacidad física, salud general, dolor y desempeño físico, así como tendencia a la depresión clínica. Al comparar dicha muestra con una muestra foránea de mujeres californianas, hubo un mejor desempeño en los índices de calidad de vida en la muestra colombiana [13].

También se encontraron 41 pacientes con buena resiliencia, afrontamiento espiritual y sistemas de creencias, así como altos puntajes en las dimensiones de vitalidad, salud general, función física y función social del cuestionario de calidad de vida. De acuerdo con la información recolectada en el estudio de Koenig, los participantes tienen una fuerte creencia religiosa, la cual se convierte en una estrategia de afrontamiento activa que les permite tener mayor autoconfianza y recuperación ante las crisis, en coherencia con la evidencia empírica disponible [14]; de igual manera, perciben una alta calidad de vida a pesar de la presencia de dolor corporal. En relación con las causas de su enfermedad, aseguraron que se debe a condiciones heredadas, pero también un buen porcentaje relacionaba la enfermedad con vivencias emocionales negativas [4].

Tanto las representaciones cognitivas como emocionales de la enfermedad misma correlacionaron fuertemente con las consecuencias negativas somáticas, sociales, emocionales, del sumario mental y económico, pues los pacientes señalaron que al contar con más recursos hay mayor certidumbre y un afrontamiento más efectivo [15]; sin embargo, se relaciona también con que a mayor comprensión de la enfermedad, hay mayor disponibilidad hacia la continuación de una vida social habitual. La medicación no necesariamente se percibe de manera positiva, pues muchas veces implica procesos de readaptación engorrosos. La resiliencia identificada permite afirmar que existe en los pacientes la aceptación de sus propias condiciones, una visión positiva de la vida y, en definitiva, una mejor adaptación [16].

En otro estudio más reciente [17] se evaluó cómo la edad, el tiempo transcurrido después del diagnóstico y el nivel educativo se asocian con la satisfacción de sesenta pacientes reumáticos; el tiempo de diagnóstico es desfavorable para la adaptación en los primeros cinco años, pero no hubo diferencias significativas en cuanto a la edad ni a los años de estudio. En ese orden, los autores sugieren que las intervenciones psicológicas son más productivas dentro de esos primeros cinco años de diagnóstico y, adicionalmente, deben incorporar aspectos como la inclusión familiar, la reorganización del entorno físico y el fortalecimiento de habilidades de afrontamiento para la prevención de eventos en salud mental y del agravamiento de la enfermedad somática.

Así mismo, una investigación descriptivacorrelacional [18] indagó por los rasgos de 
personalidad, estrategias de afrontamiento y dolor en estos pacientes y halló valores medios en relación con los factores de personalidad, excepto para las escalas de mentira y de ansiedad social (las cuales puntuaron al límite superior); por otro lado, en cuanto a las estrategias de afrontamiento más usadas, reportan la religión, la reevaluación positiva, la búsqueda de apoyo profesional, el postergar el afrontamiento (conocer más del problema y de las alternativas) y la espera. En el análisis correlacional posterior se hallaron asociaciones significativas entre evitación cognitiva con evitación emocional y comportamental, reevaluación positiva con control emocional y solución de problemas, así como espera con conformismo. Aunque los autores midieron el dolor no desconocen que la medida es subjetiva, dado que se obtuvo por autorreporte, y esta sesgada precisamente por los factores psicológicos que definen y determinan su intensidad [19].

Por otra parte, Senra y colaboradores [20] se encontraron con 103 pacientes artríticos que, comparados con el grupo control, presentaban mayor tendencia a la depresión y menores índices de calidad de vida, aun cuando ello no implique necesariamente mayor vulnerabilidad, además de diferencias significativas en los ítems de funcionamientos físico y social, roles físico y emocional, vitalidad, salud mental, dolor, salud general y tendencia a la depresión. De igual manera, en dicha investigación se reporta que la varianza de la depresión en las enfermedades reumatoides estaría predicha en un $56 \%$ por las dimensiones de vitalidad y funcionamiento social; mientras que las variables demográficas y los componentes restantes del SF-36 no se asociaron. Se comenta que tanto la condición somática como la propia depresión contribuyen a reducir la calidad de vida en salud percibida.

Un estudio similar [21] realizado con 107 pacientes señala que existe una tendencia significativa a la depresión y una baja proporción de satisfacción en el entorno laboral, lo cual contrasta con una alta satisfacción con la calidad en la cohesión y organización familiar. $\mathrm{Al}$ respecto, los autores sugieren la importancia de reconocer la depresión como una condición que se alimenta de la intensidad del dolor y del estrés vivenciado, pero que al tiempo es independiente del grado de fatiga articular, medido objetivamente.

De igual manera, una investigación descriptivo-correlacional no encontró relación estadística entre los tipos de personalidad A y C con los estilos de afrontamiento en una muestra no representativa de pacientes con artritis reumatoide [22]; tampoco existen diferencias significativas entre los estilos de afrontamiento y los tipos de personalidad evaluados en relación con la presencia/ausencia de la enfermedad.

Otro estudio [23] evaluó la disfunción sexual en mujeres con artritis reumatoide y encontró que factores como la disfuncionalidad, el dolor físico y la depresión actúan como predictores de la poca frecuencia de relaciones sexuales placenteras, aspecto que sin duda afecta en gran medida su calidad de vida percibida, su satisfacción personal e, incluso, puede contribuir a manifestar posteriormente afectaciones en su estado de ánimo.

El estudio de Vinaccia y colaboradores [24] también evaluó, desde la psicología positiva, recursos como la personalidad resistente (constructo compuesto por compromiso, control y reto) y la autoeficacia con 66 sujetos. Encontró medias elevadas de personalidad resistente y de autoeficacia, en contraste con bajos niveles de incapacidad funcional; también hallaron fuertes correlaciones entre los factores compromiso, control y reto, que indican alta flexibilidad cognitiva y disposición para afrontar el cambio, así como puntajes bajos en el inventario de dolor y escasa sintomatología depresiva, aspecto que se relaciona, probablemente, con los altos niveles de autoeficacia reportado.

En ese mismo orden, una investigación desarrollada con 79 pacientes colombianos [25] encontró que la autoeficacia se relaciona negativamente con la desesperanza, la incapacidad funcional y el dolor. Al comparar esta muestra con una análoga estadounidense, se halló que en la muestra colombiana la desesperanza aprendida, la incapacidad funcional, la rigidez, la depresión, la ansiedad y el dolor son menores; mientras que es mayor 
la autoeficacia percibida, lo que le permite mayor sensación de capacidad funcional. La desesperanza detectada se relacionó más con la falta de control que con el dolor experimentado.

Dentro de lo revisado, únicamente se evidenciaron dos esfuerzos aplicados: el primero corresponde a una intervención educativa cuyo fin último fue favorecer, desde una modalidad no farmacológica, el mejoramiento de los procesos de bienestar psicosocial, de adherencia terapéutica y de motivación hacia conductas útiles en la recuperación y reducción de la sobrecarga en las articulaciones [26]. Dicha intervención partió de la identificación de conocimientos que las personas tienen sobre su enfermedad, las pautas de autocuidado y, posteriormente, la reformulación de creencias saludables. Los resultados de dicho estudio señalan que la intervención no produce una mejora significativa en la calidad de vida de los pacientes abordados, aunque se concluye que la efectividad de las acciones educativas sí se relaciona con variables de control como la comorbilidad, la edad, el estrato socioeconómico y la duración de la enfermedad [26].

Por otra parte, un programa cuasi experimental de intervención para el fortalecimiento de la resiliencia, la autotrascendencia, el estado emocional, la calidad de vida, la percepción acerca de la propia enfermedad y el apoyo social [27] mostró tanto en el postest como tres meses después cambios significativos en las dimensiones señaladas, excepto para el impacto de la enfermedad sobre las actividades cotidianas. Las explicaciones dadas por los autores van en función de resaltar el aumento de la capacidad para buscar apoyo social, resolver problemas, incrementar el autocontrol y la autoestima, así como optimizar la adaptabilidad. De igual manera, factores como preocupación, respuesta emocional, consecuencias e identidad presentaron también indicadores óptimos en relación con los obtenidos por el grupo control. A partir de los resultados se concluye que los pacientes aprendieron a resignificar de manera positiva la enfermedad reumática como "otra experiencia en la vida" [27], incrementaron la interacción social y aumentaron la cantidad de cogniciones y conductas prosociales.

\section{Lupus eritematoso sistémico}

El lupus eritematoso sistémico (LES) es una enfermedad multifactorial que se caracteriza básicamente por la creación de autoanticuerpos que pueden causar la muerte de células no inflamatorias y alterar somáticamente el funcionamiento articular, renal y cutáneo [28], así como el funcionamiento neuronal, lo cual a su vez puede llevar a trastornos de tipo cognitivo [29] y emocional (como síntomas depresivos) [30], que afectan principalmente a mujeres jóvenes en edad fértil. En cuanto a variables psicosociales estudiadas ante el diagnóstico de LES en Colombia, se hallaron las siguientes investigaciones: con un grupo de 72 sujetos con edades, tiempos de evolución y manifestaciones clínicas heterogéneas [31] se identificó que la percepción del tratamiento de la enfermedad es menos confiable en personas de alto nivel educativo; así mismo, los pacientes crónicos, de manera paradójica, logran mayor control personal, a pesar de que dichos síntomas acarreen consecuencias graves para sus vidas desde lo somático y lo emocional. Por otra parte, este mismo estudio evidencia que la autoculpa es una forma bastante recurrente de afrontamiento y de explicación de las causas de dicha enfermedad.

Otro estudio desarrollado en el 2006 [32] evidenció que en los pacientes que reportan una calidad de vida alta en las escalas físicas y psicosociales no existen niveles clínicos ni de depresión ni de ansiedad; por el contrario, se evidencian altos niveles de optimismo disposicional, lo cual se asocia directamente con mayor afrontamiento dirigido al problema y mejor ajuste social.

Por otra parte, una muestra colombiana de 152 pacientes evidenció que los hombres obtienen indicadores altos de calidad de vida relacionada con la salud, representadas en mayores niveles de salud general, salud mental, función social y vitalidad; de igual forma, el desempeño físico en este tipo de pacientes es más afectado que 
la condición emotiva, aspecto que se relaciona directamente con la percepción general de salud [33].

Otra investigación realizada con 78 pacientes [34] identificó asociaciones significativas en cuanto a la actividad lúpica y ocupación, así como se halló que la depresión y el daño orgánico (en interacción) tienen efectos significativos en la dimensión función social, la ansiedad sobre la vitalidad y el estado civil sobre el indicador de salud general. En conclusión, se identifica que los factores fisiopatológicos y sociodemográficos afectan más la calidad de vida en la esfera mental que física, en la población evaluada.

Por último, se diseñó y validó un instrumento específico para la medición de la calidad de vida relacionada con la salud y sus dimensiones, de acuerdo con la Organización Mundial de la Salud en adultos con lupus eritomatoso [35], con un coeficiente de consistencia interna bastante aceptable (alfa $=0,98$ ). Incluso, los propios autores sugieren que dicho instrumento podría ser utilizado en otros países de Latinoamérica con previa validación cultural.

Infortunadamente, no se encontraron más estudios empíricos ni artículos que relacionen abordajes desde una perspectiva psicosocial al respecto.

\section{Fibromialgia}

La fibromialgia es un síndrome que afecta la funcionalidad de todo el sistema músculoesquelético, con dolor generalizado y recurrente, en la cual, además, se desarrollan correlatos de alteraciones psicológicas como disfunción cognitiva, sueño no reparador, trastornos emocionales y dificultades de adaptación [36], que a su vez afectan de manera negativa la calidad de vida. Es una patología en la cual se observan personas que soportan estoicamente su sufrimiento, así como pacientes que simulan de manera exacerbada manifestaciones clínicas de dolor. Son ellos quienes presentan menor autoeficacia e índice de calidad de vida, al igual que mayor anhedonia, pesimismo e introversión, aspectos que complican notoriamente el posterior abordaje terapéutico [37].

En relación con la fibromialgia, un estudio revisó la fiabilidad interna, la validez discriminante y convergente/divergente de tres cuestionarios extranjeros diseñados para reportar la calidad de vida ante la vivencia de dicha enfermedad [38]. Este encontró que los instrumentos implementados (World Health Organization Quality of Life, el Fibromyalgia Impact Questionnaire y el Medical Outcome Short Form) son congruentes para medir la CV de manera válida, efectiva y confiable en el medio colombiano. Partiendo de ello [39], se midieron las dimensiones de la calidad de vida relacionada con el padecimiento de fibromialgia en 100 sujetos y se halló que los factores que, de acuerdo con el reporte, más se afectan son la actividad física y la participación social-comunitaria, evento que puede llevar a sobrepeso, discapacidad física y enfermedades cardiovasculares concomitantes. De igual forma, evalúan que entre los factores protectores asociados a la percepción de una calidad de vida alta están la escolaridad, la actividad física, la remuneración, el acceso a los servicios en salud y la facilidad para participar de procesos educativos en torno a la enfermedad.

En cuanto a la intervención no farmacológica para el tema de la depresión en fibromialgia y sus síntomas asociados, se recomiendan la autogestión, la promoción del bienestar, el ejercicio físico y la terapia cognitiva-conductual [40]. Infortunadamente, así como en el caso del LES, no se documentan más estudios empíricos ni de intervención biopsicosocial.

\section{Conclusiones}

Si bien todos los estudios revisados confirman que lo señalado en la teoría, en relación con una prevalencia más alta de mujeres que de hombres que sufren de enfermedades reumáticas, dichas investigaciones difícilmente son comparables entre sí, dado que trabajan con muestras no probabilísticas y a que existen distinciones metodológicas, relacionadas estas últimas con 
el tipo de abordaje dado para la recolección y el análisis de la información, así como la adaptación y validación de los instrumentos de medición [5]. En la misma línea, al ser la mayoría muestras a conveniencia, se sesga notoriamente las posibilidades de generalización de los resultados hacia otras poblaciones. Asimismo, y en convergencia con otros autores [35], es necesario plantear investigaciones donde de evalúen posibles rupturas psicoafectivas y efectos negativos que puedan ser consecuencia del diagnóstico y vivencia de la enfermedad; es decir, aun cuando son evidentes en muchos de los enfermos comorbilidad con síntomas como los asociados a patologías mentales de depresión, ansiedad y estrés (derivados de la dinámica psicosocial), y estos a su vez influencian notoriamente en los mecanismos fisiopatológicos de inmunosupresión al aumentar la carga alostática de las citocinas [41,42], no existe en Colombia bastante evidencia empírica que documente el abordaje de dichos procesos psicosociales, ni mucho menos, de la retroalimentación somática asociada.

La mayoría de los estudios desarrollados son descriptivos, y aun cuando han permitido tener información de referencia para orientar hacia el desarrollo de modelos particularizados de prevención e intervención biopsicosocial, se considera pertinente realizar más estudios que permitan describir, por ejemplo, las tendencias de acuerdo con clasificaciones como grupos minoritarios [21], etnias, contextura física y condiciones socioeconómicas [16], por ejemplo, en relación con sus variables psicosociales. Por otro lado, conviene realizar más estudios de índole correlacional-confirmatorio (como los desarrollados con la metodología de ecuaciones estructurales) y experimentales que permitan evidenciar posibles relaciones de interdependencia entre las variables psicosociales y los síntomas de las enfermedades reumáticas en pacientes colombianos; en otros escritos se evidencia, por ejemplo, que así como en el caso de la calidad de vida relacionada con la salud $[5,6]$, los factores cognitivos y afectivos pueden condicionar, en buena medida, un afrontamiento más adaptativo de las condiciones estresantes asociadas al diagnóstico [43,44], que a su vez conlleva la reducción de los biomarcadores del estrés, el aumento de la competencia inmune $[45,46]$ y la elección de las opciones terapéuticas pertinentes; estos últimos, a su vez, pueden incidir en un reporte de salud mental más óptimo y útil para el propio tratamiento y mantenimiento de la funcionalidad del sujeto en diferentes ámbitos.

$\mathrm{Al}$ respecto se ha identificado que las emociones positivas pueden contribuir a a) ampliar el rango de acción y de pensamiento en situaciones propias de la vivencia de la enfermedad, b) favorecer la construcción de recursos psicológicos de afrontamiento efectivo y c) aportar en la trasformación del sujeto, haciéndolo más creativo y resiliente [4]. Esto, a su vez, incide en situaciones biomédicas como una mayor resistencia a las infecciones, menores niveles de cortisol, mayor tolerancia al dolor y disminución de las interleucinas asociadas a procesos inflamatorios [4,47]; adicionalmente, incrementan en los pacientes el control en su proceso terapéutico, evento que puede contribuir notablemente a una mejor percepción de su calidad de vida, a realizar acciones promotoras de su autocuidado médico, el autocontrol para el manejo de la depresióndesesperanza aprendida, estrés y trastornos del sueño asociadas a la sensación de minusvalía [36] y a una mayor disposición en las actividades de las sesiones terapéuticas, al reducirse, por su parte, el sufrimiento. En ese orden, conviene medir variables como inteligencia emocional, autoeficacia, salud mental positiva y otras desde perspectivas positivas [41], las cuales pueden estar funcionando en interacción como predictoras de un mejor afrontamiento y altos índices de bienestar.

Así mismo, el reconocimiento de dichos factores psicosociales particulares desde modelos biopsicosocioambientales y culturales [11,21] puede ser útil para el equipo sanitario en el diseño e implementación de intervenciones dirigidas a un manejo más adecuado de la enfermedad que incluya el desarrollo de comportamiento saludables, la participación activa de su red social de apoyo y con 
unos niveles de satisfacción más altos, lo cual puede incidir notoriamente por vía epigenéticapsiconeuroinmunológica $[42,43,44,45]$ en la reducción de la morbilidad y severidad de los síntomas asociados, así como en una mayor adhesión terapéutica $[4,8]$.

Por tal razón, es muy importante promover en los sistemas de salud latinoamericanos el tratamiento psicológico basado en la evidencia [46,47], con protocolos como los implementados desde las terapias de relajación y biorretroalimentación [48], los que combinan alternativas como psicoeducación en habilidades de afrontamiento al dolor, resolución de problemas, entrenamiento asertivo, higiene del sueño, cambio atribucional y prevención de recaídas [49], hasta las que incorporan elementos de expresión emocional [35] y fortalecimiento del afecto positivo [41], acorde con los dominios somáticos, socioculturales -familiares [36] y espirituales [15], con un acompañamiento social efectivo y adecuado, sin que este último se convierta en un refuerzo para la condición de vulnerabilidad [19], como complementos del abordaje farmacológico de dichas enfermedades.

En resumen, las investigaciones basadas en la evidencia plantean que las enfermedades reumáticas inflamatorias en Colombia, como en el resto del mundo, tienen un efecto duradero en el funcionamiento físico y psicológico de los pacientes, debido a los síntomas incapacitantes y al curso impredecible de la enfermedad. En consecuencia, muchos pacientes muestran problemas de ajuste como el estado de ánimo deprimido, lo que a su vez puede influir negativamente en el resultado de su enfermedad. Factores biopsicosociales específicos han demostrado afectar este resultado. Por ejemplo, el estrés, los factores de riesgo cognitivoconductuales (como catastrofismo y evitación del dolor) y los factores de resistencia (como el optimismo y el apoyo social) influyen en la calidad de vida, los síntomas físicos del dolor y la fatiga y los marcadores inflamatorios. Las intervenciones psicológicas que abordan estos factores pueden tener efectos beneficiosos en el funcionamiento físico y psicológico [50-51].
Por otro lado, no ubicamos análisis que involucren necesariamente unas repercusiones en el espectro social en nuestro medio, entre otras cosas porque la investigación se ha limitado a describir cómo se presentan las variables de tipo psicosocial en estas patologías.

Por este motivo, se sugiere considerar el desarrollo de estudios que aborden las variables psicosociales asociadas a enfermedades reumáticas diferentes a las que han sido abordadas tradicionalmente en Colombia, con el fin de realizar las debidas comparaciones y posteriores abordajes terapéuticos [49], y adicionalmente realizar estudios de connotación longitudinal [21], dado que las investigaciones encontradas toman la información en un único momento, pero no se encuentran estudios que den cuenta de la evolución de dichas propiedades psicosociales en relación con la progresión de la enfermedad misma.

Esto hace necesario, además, el desarrollo de investigaciones que también den cuenta de las posibles consecuencias que puede tener el diagnóstico de una enfermedad reumática en la conducta social de un individuo.

\section{Referencias}

1. Anaya JM. Genes y Artritis. Rev Colomb Reumatol. 1999;6:240-50.

2. Quiceno JM, Vinaccia S. Satisfacción con la vida según la edad, escolaridad y tiempo de diagnóstico en pacientes reumáticos. Psic Salud. 2014;24(2):157-65.

3. Correale J. Compromiso del sistema nervioso en las enfermedades reumáticas. En: Neuroinmunología clínica. Buenos Aires: Editorial Médica Panamericana; 2011.

4. Quiceno JM, Vinaccia S. Artritis reumatoide: consideraciones psicobiológicas. Diversitas: Persp Psic. 2011;7(1):27-41.

5. Lemus N, Parrado R, Quintana G. Calidad de vida en el sistema de salud. Rev Colomb Reumatol. 2014;21(1):1-3.

6. Gennen R, Newman S, Bossema ER, Vriezekolk JE, Boelen PA. Psychological interventions for patients with rheumatic 
disease and anxiety or depression. Best Pract Reseach Clin Rheum. 2012;26:305-19.

7. Sánchez MJ, García JM, Valverde M, Pérez M. Enfermedad crónica: satisfacción vital y estilos de vida adaptativos. Clin Salud. 2014;25:85-93.

8. Quiceno JM, Vinaccia S. Evolución de la intervención psicológica de la artritis reumatoide. Psicología desde el Caribe. 2011;27:160-79.

9. Vinaccia S, Ramírez LA, Toro F. Desesperanza aprendida en pacientes con artritis reumatoide: análisis y evolución de un constructo. Rev Colomb Reumatol. 2001;8:443-7.

10. Vinaccia S, Cadena C, Juárez F, Contreras F, Anaya J. Relaciones entre variables sociodemográficas, incapacidad funcional, dolor y desesperanza aprendida en pacientes con diagnóstico de artritis reumatoide. Intern Jour Clin Health Psychol. 2004;4(1):91-103.

11. Vinaccia S, Tobón S, Moreno E, Cadena J, Anaya, JM. Evaluación de la calidad de vida en pacientes con diagnóstico de artritis reumatoide. Intern J Psychol Psychol Therapy. 2005;5(1):47-61.

12. Rojas-Villarraga A, Bayona J, Zuluaga N, Mejía S, Hincapie ME, Anaya JM. The impact of rheumatoid foot on disability in Colombian patients with rheumatoid arthritis. BMC Musculoeskel Dis. 2009;10:67.

13. Vinaccia S, Fernández $\mathrm{H}$, Moreno E, Padilla G. Aplicación de la versión española del cuestionario Quality of LIfe Measure for Rheumatoid Arthritis (QOLRA) en Colombia. Rev Colomb Reumatol. 2006;13(4):264-70.

14. Koenig HG. Religion, spirituality, and health: the research and clinical implications. ISRN Psychiatry. 2012:278730. doi: 10.5402/2012/278730.

15. Kristenson M, Eriksen HR, Sluiter JK, Starke D, Ursin H. Psychobiological mechanims of socioeconomic differences in health. Soc Scien Med. 2004;58:1511-22.
16. Quiceno JM, Vinaccia, S. Resiliencia, percepción de enfermedad, creencia y afrontamiento espiritual religioso y calidad de vida relacionada con la salud en pacientes con diagnóstico de artritis reumatoide. Psicología desde el Caribe. 2013;30(3):590-619.

17. Cadena J, Vinaccia S, Pérez A, Rico MI, Hinjosa, R, Anaya JM. The impact of disease activity on the quality of life, mental health status and family dysfunction in Colombian patients with Rheumatoid Arthritis. J Clin Rheum. $2003 ; 9$ (3):143-50.

18. Gaviria AM, Vinaccia, S, Quiceno, JM, Martínez K, Yepes M, Echavarria, C, et al. Rasgos de personalidad, estrategias de afrontamiento y dolor en pacientes con diagnóstico de artritis reumatoide. Ter Psic. 2006;24(1):23-9.

19. Martin G, Pear, J. Modificación de la conducta: qué es y cómo aplicarla. México: Pearson Education; 2008.

20. Senra H, Rogers H, Leibach G, Alyamar ML, Plaza SL, Perrin P, Durán MA. Healthrelated quality of life and depression in a simple of Latin American adults with rheumatoid arthritis. Int J Rheum Dis. 2014;1-10.

21. Cadena J, Cadavid ME, Ocampo MV, Vélez MC, Anaya JM. Depresión y familia en pacientes con artritis reumatoide. Rev Colomb Reumatol. 2002;9(3):184-91.

22. Orozco AM, Palacios X, Corredor DC, Cuarta GP. Estilos de afrontamiento y patrones de personalidad en pacientes con artritis reumatoide: estudio descriptivo-correlacional. Cuad Hisp Psic. 2005;5(2):111-25.

23. Silva JM, Trujillo CG, Gutiérrez JM. Disfunción sexual en mujeres con artritis reumatoidea. Rev Urol Colomb. 2006;15(2):25-30.

24. Vinaccia S, Contreras F, Palacio C, Marín A, Tobón S, Moreno-San Pedro E. Disposiciones fortalecedoras: personalidad resistente y autoeficacia en pacientes con diagnóstico de artritis reumatoide. Ter Psic. 2005;23(1):15-24. 
25. Vinaccia S, Contreras F, Restrepo LM, Cadena J, Anaya JM. Autoeficacia, desesperanza aprendida e incapacidad funcional en pacientes con diagnóstico de artritis reumatoide. Int J Clin Health Psyc. 2005;5(1):129-42.

26. Escorcia ME, Osorio HJ, Pérez CA, Thulcán MC, Caballero CV, Borda M. Efectividad de una intervención educativa en pacientes con artritis reumatoide. Hospital Universidad del Norte, Barranquilla. Rev Colomb Reumatol. 2006;13(1):11-9.

27. Quiceno JM, Vinaccia S, Remor E. Programa de potenciación de la resiliencia para pacientes con artritis reumatoide. Rev Psicopat Psicol Clin. 2011;16(1):27-47.

28. Gómez-Puerta JA, Cervera R. Lupus eritematoso sistémico. Medicina \& Laboratorio. 2008;14:211-23.

29. Watson P, Storbeck J, Mattis P. Cognitive and emotional abnormalities in systemic lupus erithematosus: Evidence for amygdala dysfuntion. Neuropsychol Rev. 2012;22:252-70.

30. Karol DE, Criscione-Schreiber LG, Lin M, Clowse ME. Depressive symptoms and associated factors in systemic lupus erythematosus. Psychosomatics. 2013;54:443-50.

31. Valencia-Toro P, Mora C, Arbeláez AM, Jaimes DA, Guzmán Y, Plazas M. ¿Cuál es la percepción de enfermedad de pacientes colombianos con lupus eritomatoso sistémico? Aplicación del Revised Illness Perceptions Questionnarie (IPQ-R). Rev Colomb Reumatol. 2014;21(1):4-9.

32. Vinaccia S, Quiceno JM, Zapata C, Abad L, Pineda R, Anaya JM. Calidad de vida en pacientes con lupus eritomatoso sistémico. Rev Colom Psic. 2006;15:56-65.

33. Velásquez CJ, Yepes JJ, Ariza KL, Isaza PA, Gaviria AM, Muñoz-Grajales $\mathrm{C}$, et al. Estimación de la calidad de vida relacionada con la salud y medidas de utilidad en una población de pacientes colombianos con lupus eritomatoso sistémico. Rev Colomb Reumatol. 2013;20(4):183-94.
34. Ariza K, Isaza $\mathrm{P}$, Gaviria $\mathrm{A}$, Quiceno JM, Vinaccia S, Alvarán L. Calidad de vida relacionada con la salud, factores psicológicos y fisiopatológicos en pacientes con diagnóstico de lupus eritomatoso sistémico-LES. Ter Psic. 2010;28(1):27-36.

35. Quintana G, Muñetón G, Coral-Alvarado P, Méndez P, Molina J, Chalem P, Díaz J. Design and validation of LupusCol, an instrument for the evaluation of health-related quality of life in Colombian adult patients with systemic lupus erythematosus. Rheumatology (Oxford). 2014;54(1):104-12. doi: 10.1093/rheumatology/keu277

36. Vélez MC, Palacio C, Moreno AI, Krikorian A. Psychological and family-related facts of suffering in patiens with chronic disease. Tecn in Reg Anest Pain Manag. 2013;17:7-10.

37. Capilla P, González H, Santamaría P, Pérez MA, Casado MI. Fibromialgia: iexageración o simulación? Clin Salud. 2013;24:185-95.

38. Cardona-Arias JA, Hernández-Petro AM, León-Mira V. Validez, fiabilidad y consistencia interna de tres instrumentos de medición de calidad de vida relacionada con la salud en personas con fibromialgia, Colombia. Rev Colomb Reumatol. 2014;21(2):57-64.

39. Cardona-Arias JA, León-Mira V, CardonaTapias AA. Estado de salud y calidad de vida en pacientes con fibromialgia, Medellín. Rev Colomb Reumatol. 2014;21(1):10-20.

40. Millán A, Rojas MI. Evidencia del uso de antidepresivos en fibromialgia. Rev Méd Sanitas. 2010;13(2):20-6.

41. Steptoe A, Dockray S, Wardle J. Positive affect and psychobiological processes relevant to health. J Personality. 2009;77(6):1747-76.

42. Mathews HL, Witek L. Epigenetics and psychoneuroimmunology: Mechanism and models. Brain Behav Inmun. 2011;25:25-39. 
43. Kubo C, Chida Y. Psychoneuroimmunology of the mind and body. Intern Cong Series. 2006;1287:5-11.

44. Zachariae R. Psychonuroimmunology: a bio-psycho-social approach to health and disease. Scand J Psychol. 2009;50:645-51.

45. Lutgendorf SK, Constanzo ES. Psychoneuroimmunology and health psychology: An integrative model. Brain Behav Inmun. 2003;17:225-32.

46. Londoño C, Valencia SR. La investigación en el proceso de soporte empírico de las terapias: alcances y limitaciones. Act $\mathrm{Col}$ Psic. 2005;13:163-81.

47. Morales MA, Mejía JM, Reyes E. Síndrome fibromiálgico: tratamiento multidisciplinario según evidencia. Rev Colomb Reumatol. 2010;17(4):231-44.

48. Glombieswki JA, Sawyer AT, Gutermann J, Koening K, Rief W, Hofmann SG. Psychological treatments for fibromialgia: a meta-analysis. Pain. 2010;151:280-95.

49. Willams DA. Psychological and behavioral therapies in fibromyalgia and related síndromes. Best Pract Reseach Clin Rheum. 2003;17(4):649-66.

50. Graninger, M. Behavioral training as additional therapy Approach for rheumatoid arthritis. Z Rheumatol.2015;74(7):579-83.

51. Van Middendorp H, Evers, A. The role of Psychological factors in inflammatory rheumamatics diseases: from burden to tailored treatment. Clin Rheumatol. 2016;30(5):932-45.

\section{Notas}

* Conflicto de intereses: ninguno. 